Motricidade

2016, vol. 12, n. 4, pp. 1-3
(C) Edições Desafio Singular

http://dx.doi.org/10.6063/motricidade.11883

\title{
Five years in Sports Sciences
}

\author{
Mário C. Marques*
}

EDITORIAL

I was glad to read today, on December 16th of 2016, that the Sports Sciences Department of the University of Beira Interior (UBI) appears in an outstanding place in the Shanghai Ranking's Global Ranking for Sport Science Schools and Departments. We could indicate that this small department is, actually, in the top 80 of the sports schools of the world, which is something that should be highlighted.

In 2007, the head of the Department of Sports Sciences at UBI and currently president of the Faculty of Social Sciences and Humanities (UBI) called for the development of internal research in the field of sports sciences, not only on the national panorama, but above all on an international level. This wish was strongly based on a complete lack of research in the department, in which until 2007 there was no single publication with indexing, much less with ISI or Scopus or any relevant project or book recognized by the scientific community. It was a herculean and risky challenge, but today we think that it has been more than achieved. At that time, the cornerstones for the development and sustainability of the investigation in the department were based on three key-vectors: selection and recruitment of more teachers with experience and research potential; acquisition of equipment, laboratory software and reformulation of the advanced training proposal (reformulation of the Master course and Doctoral study plan).

As a complement to the quality of the existing teachers, external professors were hired. The rectory was present in the creation of an R \& D unit in consortium with UTAD, UMa and 5 Polytechnics Schools, under the designation of CIDESD with headquarters in UTAD and a center in UBI. As far as the equipment is concerned, the department has acquired high quality material and diversification through internal funds (Department/Faculty) and also with the individual scientific production funds of the research unit CIDESD/UBI. Between 2008 and 2013, these acquisitions encouraged the exponential writing of articles, the development of projects and the conclusion with high quality of masters and doctoral theses. This was only possible due to the high scientific production carried out, which was strictly thought in favor of our students and the Department of Sports Sciences. The acquisition of research material had not only a typical laboratory concern but also an ecological one, that is, most of the acquired equipment was portable, allowing us to carry out several studies outside the Department, going to the places where the individuals that composed the samples were. Finally, since 2009, there has been a strong internationalization policy for the Masters course, especially with the arrival of highly qualified teachers from worldwide, which has allowed the promotion of research policies and a significant increase in quantity, but above all in the quality of the published articles. In this research policies project - it was sought to present a line of concrete study that addressed some pertinent problems to which the international literature has not yet shown any final conclusions.

In the last two decades, scientific research in Sport Sciences has grown exponentially in the department. Unfortunately, most of the resources, such as critical mass or equipment (laboratories) are still scarce in our country, although some universities have taken important steps in order to reverse this situation. Thus, UBI could not remain unaware of this "revolution". We had a young department with quality and capacity to perform quality research. To this purpose, it was urgent to develop protocols and / or connect with universities and researchers of international reference, which would transport us to higher levels of research. Since our field of study was so vast and complex, we had to focus on the following points of interest: A) preparing research projects in the field of sports performance; B) drawing short - term strategies for the construction of a

\footnotetext{
* Corresponding author: Departamento de Ciências do Desporto da UBI, Rua Marquês Ávila e Bolama, 6201-001, Covilhã, Portugal.E-mail: mmarques@ubi.pt
} 
root laboratory that would be able to transport us to the "front line"; C) helping integrate our young doctoral students (teachers) into the "world" of research. Considering the opening of the European university space resulting from the Maastricht Treaty, one of the pillars of the internationalization policy has been the focus on European cooperation activities. Numerous protocols were developed with the University of Pitesti, the Public University of Navarra, the Pablo de Olavide University, and the University of Barry State. These contacts were a result from the social and academic networks established with members of these universities.

After hiring the new professors whose doctorates were concluded between 2007 and 2009, the mission of equating a course development strategy and improving its attractiveness was crucial. Given the fact that, at the level of the 2nd cycle offer, the demand was low, it was therefore necessary to attract students from other schools of the country. It was obvious that this would only be possible with the use of previous personal knowledge networks and the support of the Center for Research in Sport, Health and Human Development (CIDESD), a research center where UBI is an integrated member.

The 2nd cycle of studies of the Master's Degree in Sports Sciences was created in the 1st year of the Bologna Process adjustments of the courses given at the UBI. At that time, the Department's doctoral faculty was exiguous and very little diversified. For this reason, the Curricular Units proposed for the curriculum were based in the possibility of hiring other human resources. National and international teachers of recognized pedagogical and scientific value were recruited, with special emphasis on the prestigious curriculum of publications in the area of Sports Sciences. Provisional calls were launched and readily accepted on the condition that they taught concentrated classes, similar to what already being done in many foreign universities and also in some national ones.

In the main scientific area of the cycle of studies (Sports Sciences) all the teachers integrated in the service distribution are effective members or collaborators of CIDESD. CIDESD is a research unit accredited by FCT (since the $12^{\text {th }}$ of December of 2009) with the initial classification of GOOD and nowadays of VERY
GOOD. Also, worth mentioning, is the collaboration with the Center for Excellence in Studies, Research and Sports Medicine and the Navarro Institute of Sport, Government of Navarra.

The approach to scientific research has also been a point of honor of this department, carried out in a sustainable way, mainly through teaching/learning methodologies specific to each curricular unit (CU), mostly through research seminars. This approach begins in the 1st semester of the 1st year, encouraging the student to the good practices of scientific research, particularly in his area of interest. However, the ultimate milestone of his effective integration into the scientific research can only be consolidated if the student is qualified to prepare or eventually to submit a scientific paper in an ISI-indexed journal provided by the Seminar CUs. Finally, we must highlight the involvement in the implementation of technical-scientific events allowing contact with basic and applied science, of which the Research Seminar of CIDESD and CIDESD Junior is the best example. It should also be said that the scientific activity produced by teachers and students is strongly implemented in the methodological orientation of teaching / research and in the provision of services and advice to the academic community and to civil society in general. Regarding to the research-community relationship, the type of research developed is powerfully applied by integrating and transmitting immediately the produced knowledge to the stakeholders (e.g., clubs, municipalities, gymnasiums and swimming pools). Therefore, this applied research par excellence in the physical activity context of exercise and sport in its most diverse fields of application brings economic benefits to the partners of the course.

It should be mentioned that in the last two years there has been a significant increase in the publication of scientific articles in journals indexed to the ISI Web of Knowledge, a true and successful Case Study at the national level. Also note that part of the articles published during the last years were launched in magazines with an impact factor higher than 1.o. Also noteworthy are the publications in book or chapter format of books with scientific review. There are also dozens of abstracts published in national and international conferences (with scientific review). 
In fact, we consider this type of publication as an excellent measure of dissemination of the work produced by senior researchers and 3rd cycle students. In some cases, even for the 2nd cycle students.

We succeeded in spreading knowledge through the range of articles available in worldwide renowned journals, i.e.: Original Research, Brief Reviews, Reviews, Methodological Reports, Research Notes, and Letters to Editor. In terms of impact, if we consider that the UBI Teaching Activity Regulation defined $\mathbf{0 . 4}$ as the impact reference median to the Sports Sciences, the publications in question are clearly above this level with an average close to 1.0 impact, a high value for the sports sciences. It should be emphasized that more than 50 percent of the articles refer to 1.8-1.9 impact journals, and that we have had a review - recently published in the highest impact factor journal of the area (Sports Medicine: 5.2).

With this philosophy of publications, it was intended to carry out a large number of scientific studies that addressed a panoply of issues considered more relevant like the ones related to the effectiveness of Strength Training and Physical Condition on performance improvements in High Performance Sports, Public School and Exercise /Health. Consequently, this line of thought / intervention, in addition to discussing in a pragmatic and scientific way different topics related to the methodology of Strength Training and Physical Condition, tried to do a parallelism between theory and practice, that is, most of the abovementioned articles are of a highly practical nature in order to daily assist coaches, physical education teachers and health/sport professionals. We also analyzed the Simultaneous Training of Strength and Aerobic thematic, as well as the problematic of the Detraining. These are two hot topics as both are far from consensus in the scientific community.

Since the origin of the Department (1994), the first four experimental studies conducted in our laboratories have been published in two of the best sports training magazines. In the five-year period in question, the level of scientific production was exponential with more 100 international ISI articles published or accepted for publication in journals indexed to international reference databases by the end of 2013. The participation in conferences such as the American College of Sports Medicine (ACSM) and the European College of Sport Science (ECSS) as well as the positive evaluation of $2 \mathrm{R} \& \mathrm{D}$ projects by international panels (with emphasis on the project approved in call 2010) seem to indicate that the scientific community recognizes the efforts done to contribute for a better understanding of the sportive phenomenon, both in theoretical as in empirical terms. We should also note the level of involvement in the scientific community with referee reports for international reference journals and with several coauthors affiliated to different universities (national and international).

The research networks developed in 5 years and the funding of the international $\mathrm{R} \& \mathrm{D}$ Projects planned for the coming years, will not only allow the renewal of equipment and software, but also bring the possibility of hiring highly qualified human resources, guaranteeing important conditions to continue in the line of international merit investigation. It is also an important incentive to further progress in the worldwide scientific production, recognized by the scientific community as well as helping UBI to consolidate its role in the country and in the world, in this scientific area. However, there are still some teachers who feel some lack of motivation to publish regularly. 